\title{
Electron acceleration in a wavy shock front
}

\author{
M. Vandas and M. Karlický \\ Astronomical Institute of the Academy of Sciences of the Czech Republic, 25165 Ondřejov, Czech Republic
}

e-mail:vandas@ig.cas.cz

Received 12 November 2010 / Accepted 22 April 2011

\begin{abstract}
Context. It is known that electrons are accelerated at nearly perpendicular shocks by the drift mechanism. And it is also known that energy gain of electrons caused by this mechanism is not very high. Therefore it was suggested in the past that the energy gain might be increased if shocks had wavy fronts. For instance, there were attempts to explain coronal type II burst and their fine structure by electron acceleration in a wavy shock front.

Aims. We studied electron acceleration numerically at nearly perpendicular wavy shocks for coronal conditions and compared it with analytical results on electron acceleration at nearly perpendicular plane shocks.

Methods. An analytical model of a wavy shock front was used and trajectories of electrons in it and around it were calculated numerically in a guiding centre approximation.

Results. We found that energy gains of electrons at a wavy shock front and a corresponding smoothed-into-plane shock on the average were comparable. That is why they do not depend significantly on the shock thickness, magnetic field profile inside the shock, and shock wavy form. They do depend on the angle between the smoothed shock front and ambient magnetic field.

Conclusions. On average, a wavy shock front does not significantly increase an acceleration efficiency. Energy gain remarkably exceeds an average level for some combinations of initial parameters. Distribution functions of accelerated electrons have a patchy structure, which is prone to inducing plasma instabilities that will generate plasma waves. This may have relevance to the problem of type II burst origin.
\end{abstract}

Key words. acceleration of particles - shock waves - magnetic fields - Sun: radio radiation

\section{Introduction}

Holman \& Pesses (1983) suggested that electrons were promptly accelerated by quasi-perpendicular shocks in the solar corona. Theory of electron acceleration by nearly perpendicular plane shocks was formulated by Wu (1984) and Leroy \& Mangeney (1984) and labelled as a fast Fermi process. Vandas (1989) and Krauss-Varban \& Wu (1989) identified this process as a shock drift mechanism inside the shock layer. Energy gain of electrons during the process is not high, only several times the initial energy, therefore it was suggested that a wavy shock front could enable electrons to interact more times with the shock and so gain more energy (e.g., Zlobec et al. 1993). In this paper we examine this possibility quantitatively.

Influence of ripples in a shock wave front on particle acceleration has also been investigated by Decker (1990) and Gedalin (2001), but their studies are not directly applicable to our problem. The former studied a large-scale rippling and proton acceleration, and the latter a small scale rippling and suprathermal proton and electron motion. Knocks et al. (2003) investigated the influence of rippling on interplanetary type-II burst generation by accelerated beams of electrons.

Vandas \& Karlický (2000) investigated electron acceleration by a perpendicular wavy shock. This paper is hereafter referred to as Paper I. The perpendicular geometry treated in Paper I yielded that eventually all interacting electrons had been transmitted downstream. Here we treat cases with nearly perpendicular wavy shocks where some reflected electrons may escape upstream. And it is known that reflected electrons have on average a higher energy gain than do transmitted ones.

\section{Model}

A model of a wavy shock is shown in Fig. 1, and it is the same as in Paper I. The shock front is formed by a sinusoidal wave with the wavelength $L$ and amplitude $A$ (right part of the figure, plotted size of $A$ is exaggerated). Upstream magnetic field lines were parallel to the $Z$ axis (parallel to a smoothed shock front, a perpendicular shock) in Paper I. In the present analysis a small inclination of the field lines is allowed (a nearly perpendicular shock). Using a procedure described in Vandas (1995) and following Paper I, the wavy shock front is converted into a plane shock wave with the thickness $d$ and $B_{\mathrm{n}}$ varying (left part of Fig. 1). $B_{\mathrm{n}}$ is the normal component of the upstream magnetic field with respect to the shock front, and its variation is specified later, in Eqs. (13) and (15). Electron motion in the guiding centre approximation inside and outside the shock layer is then calculated.

The shock front is defined by the equation

$X+A(1-\cos \omega Z)=0$

with

$\omega=2 \pi / L$

The orientations of the $X$ and $Z$ axes are shown in Fig. 1 . 


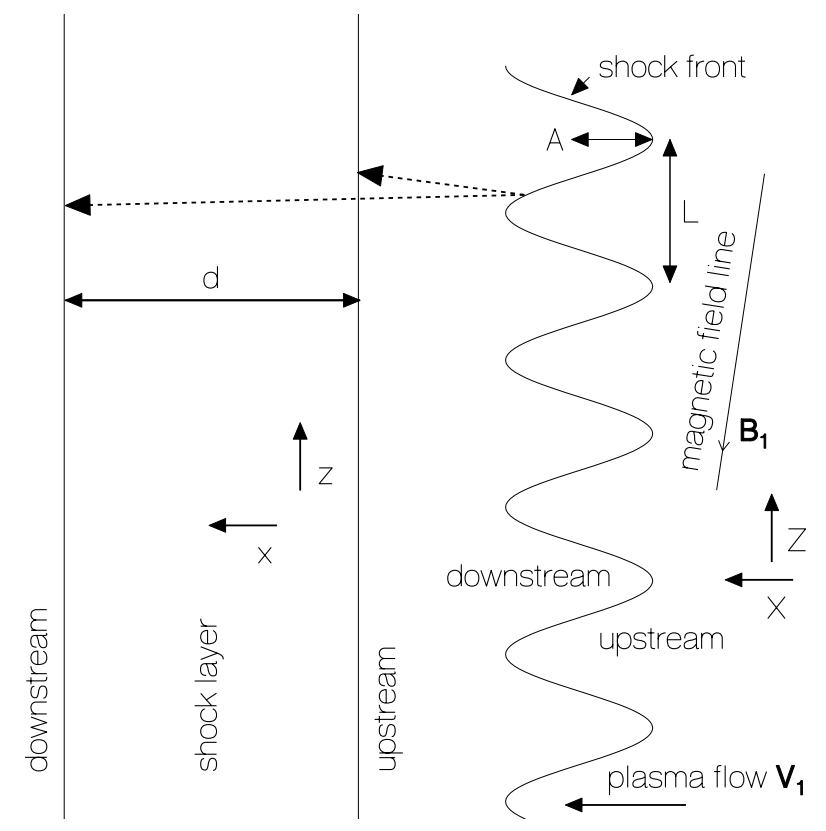

Fig. 1. Global view of the model wavy shock front on the right. Left part shows a wavy shock approximation of a magnified small piece of the wavy shock (see two dashed arrows) by a plane shock wave. $X$ and $Z$ scales are not the same, the $X$ scale is zoomed in.

Electrons upstream and downstream are assumed to move in homogeneous fields, which is a simplification of a real situation. The upstream magnetic field in the $X Y Z$ system approximately is

$\boldsymbol{B}_{1}=\left(B_{1} \cos \theta_{B}, 0,-B_{1}\right)$,

where $B_{1}=$ const. is the upstream magnetic field magnitude and $\theta_{B}=$ const. is the angle between the upstream magnetic field vector and a normal to the smoothed shock front. (This angle is usually denoted as $\theta_{B_{\mathrm{n}}}$ for a plane shock wave, but $\theta_{B_{\mathrm{n}}}$ varies for a curved shock wave.) Paper I treated the case with $\theta_{B}=$ $90^{\circ}$. Here, for a nearly perpendicular shock it is assumed that $\theta_{B}$ is close to $90^{\circ}$; therefore, we set $\sin \theta_{B} \approx 1$. The downstream magnetic field is supposed to be

$\boldsymbol{B}_{2}=\left(B_{1} \cos \theta_{B}, 0,-v B_{1}\right)$,

where $v$ is a shock magnetic-field jump. The upstream plasma velocity is

$\boldsymbol{V}_{1}=\left(V_{1}, 0,0\right)$,

and the electric field is assumed to be constant everywhere,

$\boldsymbol{E}=-\boldsymbol{V}_{1} \times \boldsymbol{B}_{1}$.

In the shock layer, electron motion is governed by equations in the guiding centre approximation (Vandas 1995, Paper I)

$\dot{v}_{\|}=-\frac{\mu}{m} B_{1} h \frac{q^{\prime}}{q}$

$\dot{x}=\frac{1}{q}\left(v_{\|} h+V_{1}\right)$,

$\dot{y}=\frac{\mu}{e} \frac{q^{\prime}}{q}$

$\dot{z}=-v_{\|}$,

$\dot{E}_{\mathrm{k}}=\mu V_{1} B_{1} \frac{q^{\prime}}{q}$, where $m$ is the electron mass, $e$ the elementary charge, $\mu$ the electron magnetic moment, $E_{\mathrm{k}}$ the electron kinetic energy, $v_{\|}$the electron velocity along the magnetic field line, $V_{1}$ and $B_{1}$ (already introduced) are upstream plasma velocity and magnetic field, respectively. Dots denote time derivatives, and prime denotes derivatives by $x$. The equations are expressed in the system $x y z$ of the transformed plane shock, which is different from the system $X Y Z$ (see Fig. 1). The system $x y z$ is only used inside the model plane shock, and it holds $x \| X, y \equiv Y$, and $z \equiv Z$, while $x=0$ and $x=d$ describe the upstream and downstream edges of the shock layer, respectively.

The functions $h$ and $q$ define magnetic field inside the shock layer $(0 \leq x \leq d)$,

$\boldsymbol{B}=\left(B_{1} h(z), 0,-B_{1} q(x)\right)$,

so the function $h(z)$ is related to the $B_{\mathrm{n}}$ component by

$B_{\mathrm{n}}(z)=-B_{1} h(z)$.

Because

$B_{\mathrm{n}}=\boldsymbol{B}_{1} \cdot \boldsymbol{n}$

where $\boldsymbol{n}$ pointing upstream is a unit vector to the shock surface (1), it follows from (13), (3), and (1) that

$h(z)=\cos \theta_{B}-A \omega \sin \omega z$.

The function $q(x)$ is related to a magnetic field profile in the shock layer and it holds $q(0)=1$ and $q(d)=v$. Paper I, in accordance with Vandas (1989), used the following expression for $q$,

$q(x)=\exp (x \ln v / d)$.

It enabled them to solve the Eqs. (7)-(11) analytically. However, for a more complex $h$ like (15), one can proceed analytically only half way. It has been shown by Vandas \& Karlický (2005) (hereafter referred as Paper II), which contains preliminary results on electron acceleration by a nearly perpendicular wavy shock. Therefore a numerical treatment is necessary. It enabled abandoning the profile (16), which is somewhat artificial (Fig. 2), and unlike Paper II to use a more realistic profile (Vandas 1991)

$q(x)=1+\tau(1-\cos \lambda x)$

with

$\lambda=\pi / d, \quad \tau=(v-1) / 2$.

The profile is shown in Fig. 2.

\section{Results}

The model shock parameters used in our numerical calculations were $B_{1}=0.5 \mathrm{mT}(5 \mathrm{G}), V_{1}=1000 \mathrm{~km} \mathrm{~s}^{-1}, v=1.6$, and $d=50 \mathrm{~m}$. The calculations showed that results were nearly the same for the same ratios $A / L$. Calculations are presented for the ratios of 0.1 and 0.2 with $L=1000 \mathrm{~km}$. These parameters (corresponding to a coronal shock) were the same as used in Papers I and II, because it was our intention to have a direct comparison with their results. Calculations are presented for $A / L=0.1$ if not said otherwise. 
M. Vandas and M. Karlický: Electron acceleration in a wavy shock front

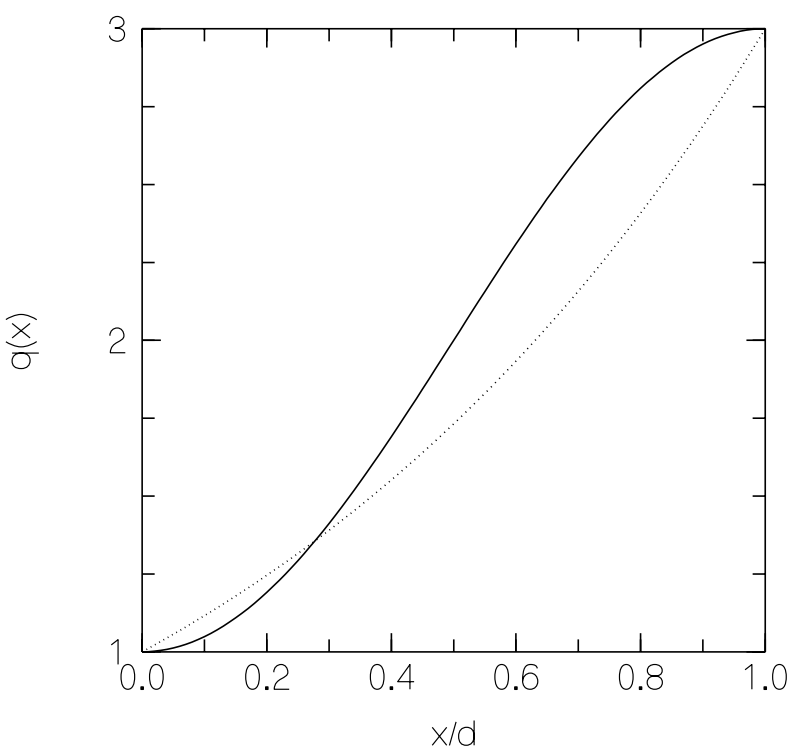

Fig. 2. Magnetic field profiles inside the model shock layer. The profile (17) used in calculations (solid line) is compared to the profile (16) drawn by the dotted line. The magnetic jump $v=3$ was used in the plot.
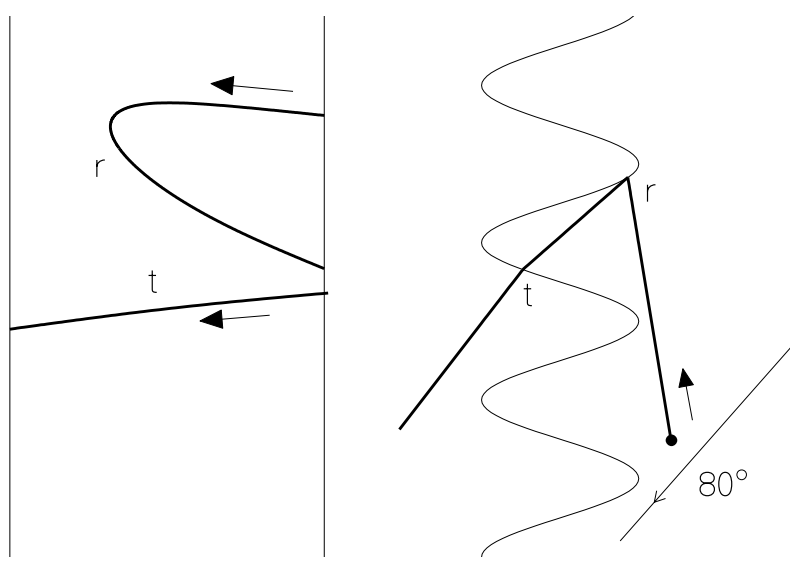

Fig. 3. Example of an electron trajectory around (right) and inside (left) the wavy shock. The electron is reflected (r) once and then transmitted $(\mathrm{t})$ downstream. Relative vertical placement of $\mathrm{r}$ - and $\mathrm{t}$-trajectories is arbitrary (otherwise they would not fit both into the figure). A value of the angle $\theta_{B}$ is explicitly written.

\subsection{Calculations of electron trajectories}

For electron trajectory determination, the Eqs. (7)-(11) were solved numerically inside the shock layer and electron moved in homogeneous magnetic fields outside the layer. Figure 3 shows an example of an electron trajectory around and in the wavy shock with $\theta_{B}=80^{\circ}$. The layout is similar to Fig. 1 . The electron trajectory is drawn by the thick line. Horizontal ( $X$ and $x$ ) directions are zoomed in comparison with vertical $(Z$ and $z$ ) directions, and the $x$ direction is much more zoomed in than the $X$ direction. The electron with initial energy $E_{i}=1 \mathrm{keV}$ and pitch angle $\alpha_{i}=105^{\circ}$ is reflected once and then transmitted, and its final energy is $E_{f}=2.1 \mathrm{keV}$ (" $i$ " and " $f$ " are used for initial and final values, respectively). For comparison, the same electron interacting with a corresponding smoothed shock wave (i.e., the wavy shock smoothed into a plane shock wave) with $\theta_{B_{\mathrm{n}}}=80^{\circ}$ would be transmitted and its final energy would only be $1.1 \mathrm{keV}$. The pitch angle is the angle between the velocity and
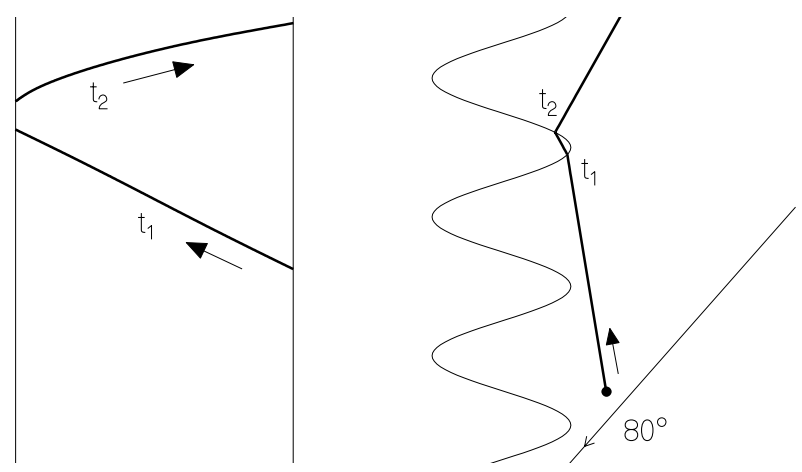

Fig. 4. Another example of an electron trajectory around (right) and inside (left) the wavy shock. Layout is similar to Fig. 3. The electron only changed its initial position and it is one time transmitted ( $t$ ) downstream and one time transmitted (t) upstream.
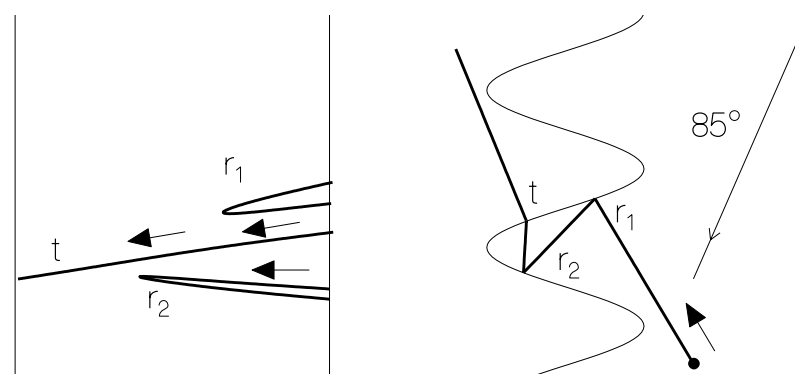

Fig. 5. Example of an electron trajectory around (right) and inside (left) the wavy shock. Layout is similar to Fig. 3. The angle $\theta_{B}$ only changed. The electron is reflected $\left(\mathrm{r}_{1}, \mathrm{r}_{2}\right)$ twice and then transmitted $(\mathrm{t})$ downstream.

magnetic field vectors, and the pitch angle $0^{\circ}$ has an upstream electron moving along the magnetic field towards the shock.

If the initial position of the electron from Fig. 3 is shifted along $Z$ by approximately $L / 3$, we get a trajectory shown in Fig. 4. The electron is transmitted downstream and then transmitted upstream, with a final energy of $1.7 \mathrm{keV}$. Overall, the electron can be regarded as reflected. Electron trajectory and its energy gain are quite sensitive to electron position with respect to a wavy shock front. For both cases the energy gain is higher than on the corresponding smoothed shock wave. However, this is not a rule.

Figure 5 shows an electron trajectory of the same electron as in Fig. 3 but for a shock with $\theta_{B}=85^{\circ}$. This electron interacting with a smoothed shock wave would be transmitted and its final energy be $1.9 \mathrm{keV}$. Even though the electron is reflected twice on the wavy shock front and then transmitted, its final energy is only $1.6 \mathrm{keV}$, i.e., lower. Electron acceleration is very sensitive to $\theta_{B_{\mathrm{n}}}$, so not only does number of interactions play a role, but also where on the wavy shock an electron interacts.

Calculations were made with the magnetic field profile (17). Similar calculations but with the magnetic field profile (16) were presented in Paper II. If we compare Fig. 3 here with Fig. 3 in Paper II or Fig. 5 here with Fig. 4 in Paper II, we see that trajectories are very similar and energy gains practically the same. This confirms conclusions by Vandas $(1991,1995)$ that energy gain does not significantly depend on a magnetic field profile in a curved shock (cf. for a plane shock wave, where the gain does not depend on it at all).

Calculations show that electron trajectories and energy gains depend very much on $\theta_{B}$, electron initial energy, pitch angle, and position. Sometimes electrons gain more energy than at 


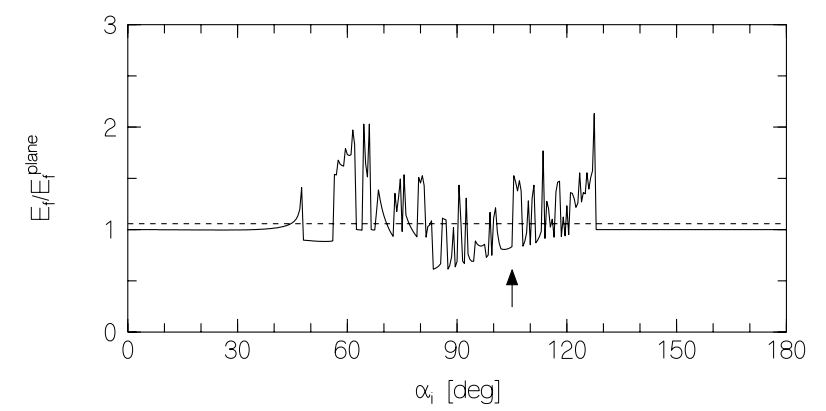

Fig. 6. Comparison of energy gain of electrons at the wavy shock with one at a smoothed shock. $E_{f}$ and $E_{f}^{\text {plane }}$ are final energies after interactions with the wavy shock and a smoothed shock, respectively, $\alpha_{i}$ is the initial pitch angle. The dashed line displays an average value.

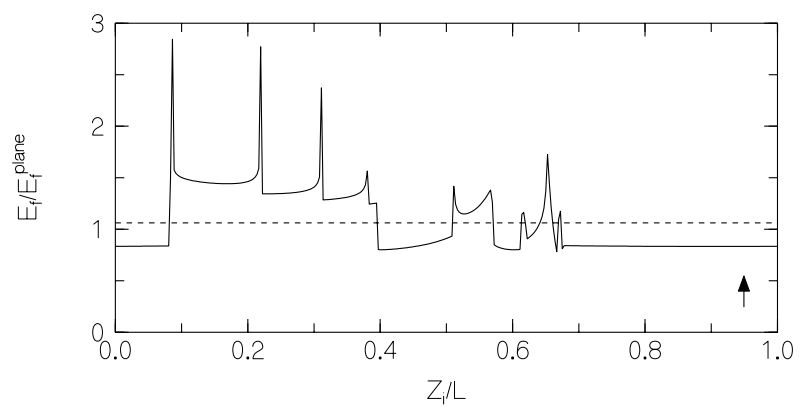

Fig. 7. Comparison of energy gain of electrons at the wavy shock with one at a smoothed shock. $E_{f}$ and $E_{f}^{\text {plane }}$ are final energies after interactions with the wavy shock and a smoothed shock, respectively, $Z_{i}$ is the initial position. The dashed line displays an average value.

a corresponding plane shock, sometimes less. To obtain a clearer picture, we calculated dependencies of energy gains on some initial parameters.

We started with the situation shown in Fig. 5. The shock had $\theta_{B}=85^{\circ}$. The electron with initial energy $E_{i}=1 \mathrm{keV}$ and pitch angle $\alpha_{i}=105^{\circ}$ was released from the place with $X_{i}$ and $Z_{i}$. Its final energy was $E_{f}=1.6 \mathrm{keV}$. At a smoothed shock wave, its final energy would be $E_{f}^{\text {plane }}=1.9 \mathrm{keV}$. Now we change $\alpha_{i}$ (Fig. 6) or $Z_{i}$ (Fig. 7) and compare energy gains on the wavy shock and a smoothed shock wave. The other parameters are kept constant. Figures 6 and 7 display this comparison, and arrows indicate the case from Fig. 5. We see frequent jumps up and down from 1, which reflects a sensitivity to initial parameters. In maxima the final energy for the wavy shock does not exceed three times the final energy at a corresponding smoothed shock. The average values are close to 1 . It means that energy gains at a wavy shock and a smoothed shock are comparable on average. But for some "preferred" or "resonant" initial parameters, the energy gain at a wavy shock may be significantly larger.

The above-mentioned sensitivity can be explained by complex interactions with the wavy shock. Figure 8 shows extreme examples, electrons, which are reflected and then transmitted downstream and upstream several times. They end upstream, so from a global point of view, they are regarded as reflected. Figure 8a plots a trajectory of an electron with the initial energy $1 \mathrm{keV}$ and pitch angle $65^{\circ}$. The final energy was $2.3 \mathrm{keV}$. The same electron interacting with a smoothed shock wave would be transmitted and its final energy would be $1.3 \mathrm{keV}$. It is interesting to note that, despite this complex interaction, calculations with the different magnetic field profile (16) yield nearly the same
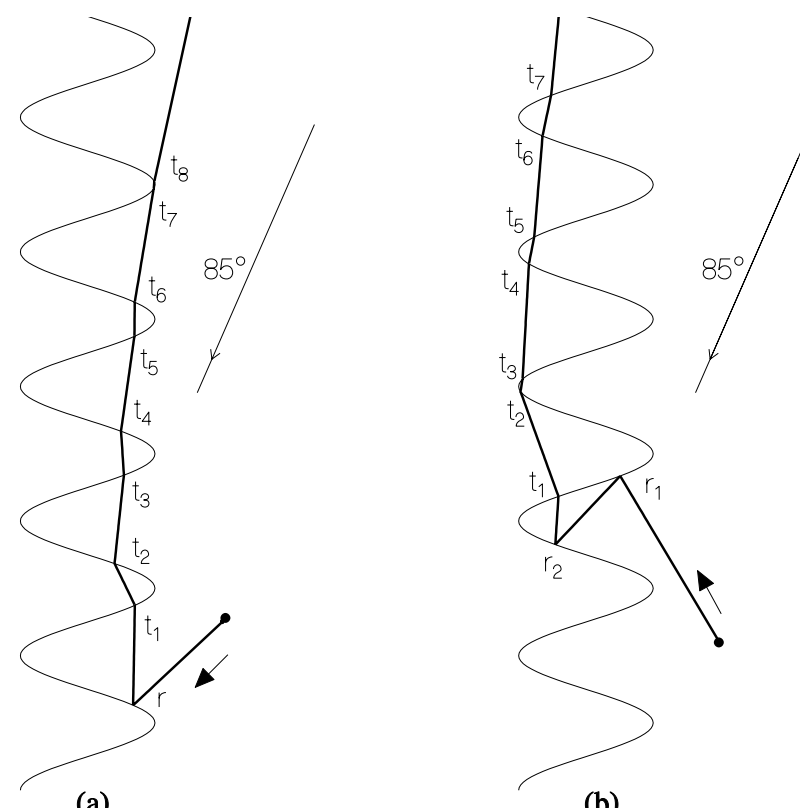

Fig. 8. Examples of electron trajectories around the wavy shock. a) The electron is reflected ( $r$ ) and then transmitted many times $\left(t_{1}-t_{8}\right)$ upstream/downstream and vice versa. b) A similar trajectory but for a higher energy gain. The electron is reflected twice $\left(r_{1}\right.$ and $\left.r_{2}\right)$ and then transmitted many times $\left(\mathrm{t}_{1}, \mathrm{t}_{2}\right.$, etc. $)$.

trajectory and energy gain (cf. Fig. 5 in Paper II). Figure 8b displays the case of the first maximum in Fig. 7.

Sensitivity of the solution to initial parameters raises a question of computational accuracy. We examined this matter carefully. Paper I presents an analytical solution of electron trajectories for the shock magnetic field profile specified by (16) and $\theta_{B}=90^{\circ}$. Comparison of this solution with numerical calculations used in the present paper yielded practically identical results. Numerical calculations of electron trajectories for cases where an analytical solution is absent (as in this paper) were checked through changes in integration time steps by several orders of magnitude, again with practically identical results.

\subsection{Calculations with an electron distribution function}

Using the above discussed trajectory calculations (but in backward direction with respect to time) and the Liouville theorem, a distribution function of accelerated electrons can be constructed from an initial distribution function. The same initial distribution function is used as in Papers I and II. It describes halo electrons (suprathermal tail) by a kappa function

$f_{i}(v)=\frac{n_{\mathrm{H}} c_{\kappa}}{\pi^{3 / 2} v_{\mathrm{H}}^{3}}\left[1+\frac{v^{2}}{v_{\mathrm{H}}^{2}\left(\kappa-\frac{3}{2}\right)}\right]^{-\kappa-1}$,

where $n_{\mathrm{H}}$ is the halo density, $v_{\mathrm{H}}$ the characteristic halo electron velocity,

$c_{\kappa}=\frac{\Gamma(\kappa+1)}{\Gamma\left(\kappa-\frac{1}{2}\right)\left(\kappa-\frac{3}{2}\right)^{3 / 2}}$,

$\Gamma$ is the gamma function, and $\kappa$ determines the deviation of the halo distribution from a Maxwellian one. It is set $\frac{1}{2} m v_{\mathrm{H}}^{2}=0.6 \mathrm{keV}$ and $\kappa=6$ (as in Papers I and II, see discussion in Paper I), and value of $n_{\mathrm{H}}$ is not necessary for the present calculations. 

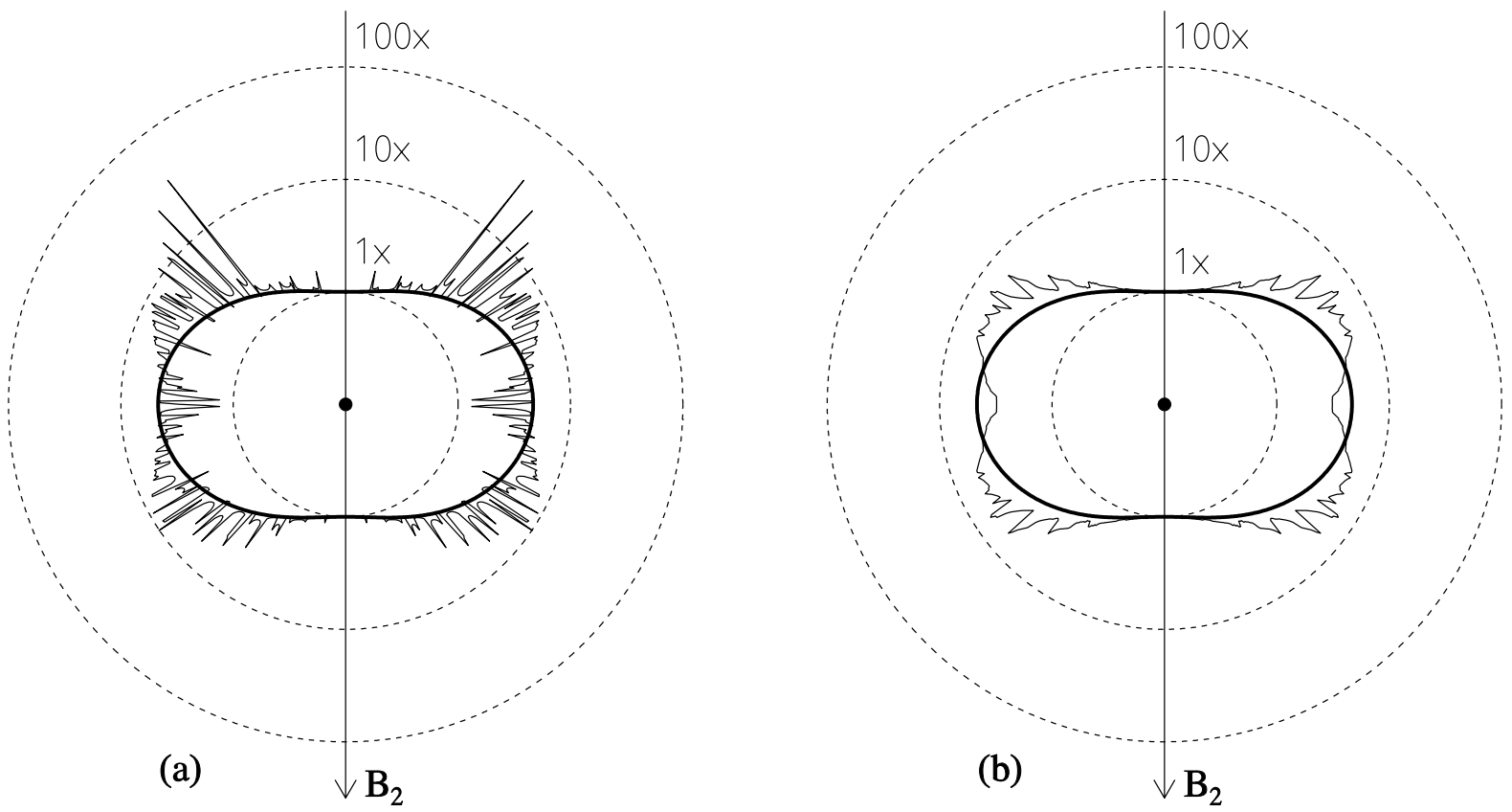

Fig. 9. a) Angular distribution function of accelerated downstream electrons for a perpendicular wavy shock in an arbitrarily chosen downstream point (thin solid line). The direction of the magnetic field $\boldsymbol{B}_{2}$ is shown. The shock is located opposite to this direction. The distribution function is rotationally symmetric around $\boldsymbol{B}_{2}$. It is a polar graph, where the polar angle corresponds to the pitch angle $\alpha$ and the radial distance (in a logarithmic scale) to a value of the distribution function $f(v, \alpha)$ of accelerated downstream electrons. The velocity $v$ is constant here and its value corresponds to the energy of $3 \mathrm{keV}$. The dashed circle labelled $1 \times$ represents the value of the initial distribution function $f_{i}(v)$ from (19). For comparison, the thick solid line shows the angular distribution function of transmitted electrons through a perpendicular plane shock wave. b) The same as in a), but the thin solid line shows the angular distribution averaged along the shock front.
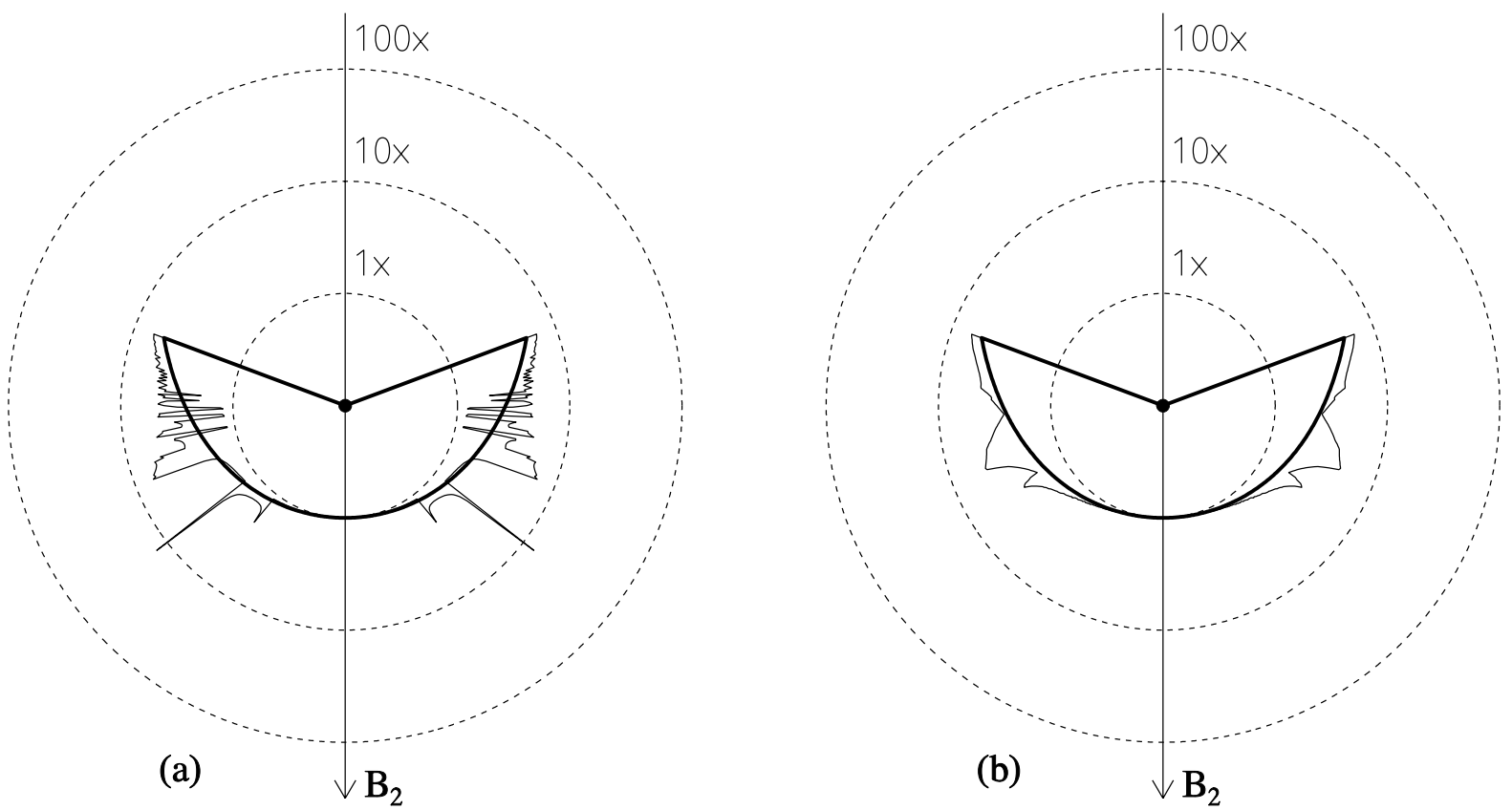

Fig. 10. a) Angular distribution function of accelerated downstream electrons with energy of $3 \mathrm{keV}$ for a wavy shock with $\theta_{B}=85^{\circ}$ in an arbitrarily chosen downstream point (thin solid line). The layout is similar to Fig. 9. The thick line shows the distribution of transmitted electrons through a smoothed shock wave with $\theta_{B_{\mathrm{n}}}=\theta_{B}$. b) The same as in a), but the thin solid line shows the angular distribution averaged along the shock front.

The next figures display angular distribution functions of accelerated electrons with respect to the magnetic field direction. All angular distribution functions presented here are for $A / L=$ 0.1. Papers I and II show some comparison with other $A / L$, but results are not very different. Figure 9a shows an angular distribution function of downstream electrons with the final energy of $3 \mathrm{keV}$ at a wavy shock with $\theta_{B}=90^{\circ}$ (i.e., with $\theta_{B}$ treated in Paper I). In this geometry all electrons finally end downstream. The distribution is for an arbitrarily chosen place with $X_{f}$ and $Z_{f}$. A spiky character reflects already discussed sensitivity to initial values. A different downstream place would yield a similar picture but with "spikes" at different $\alpha$ 's and with different sizes. 

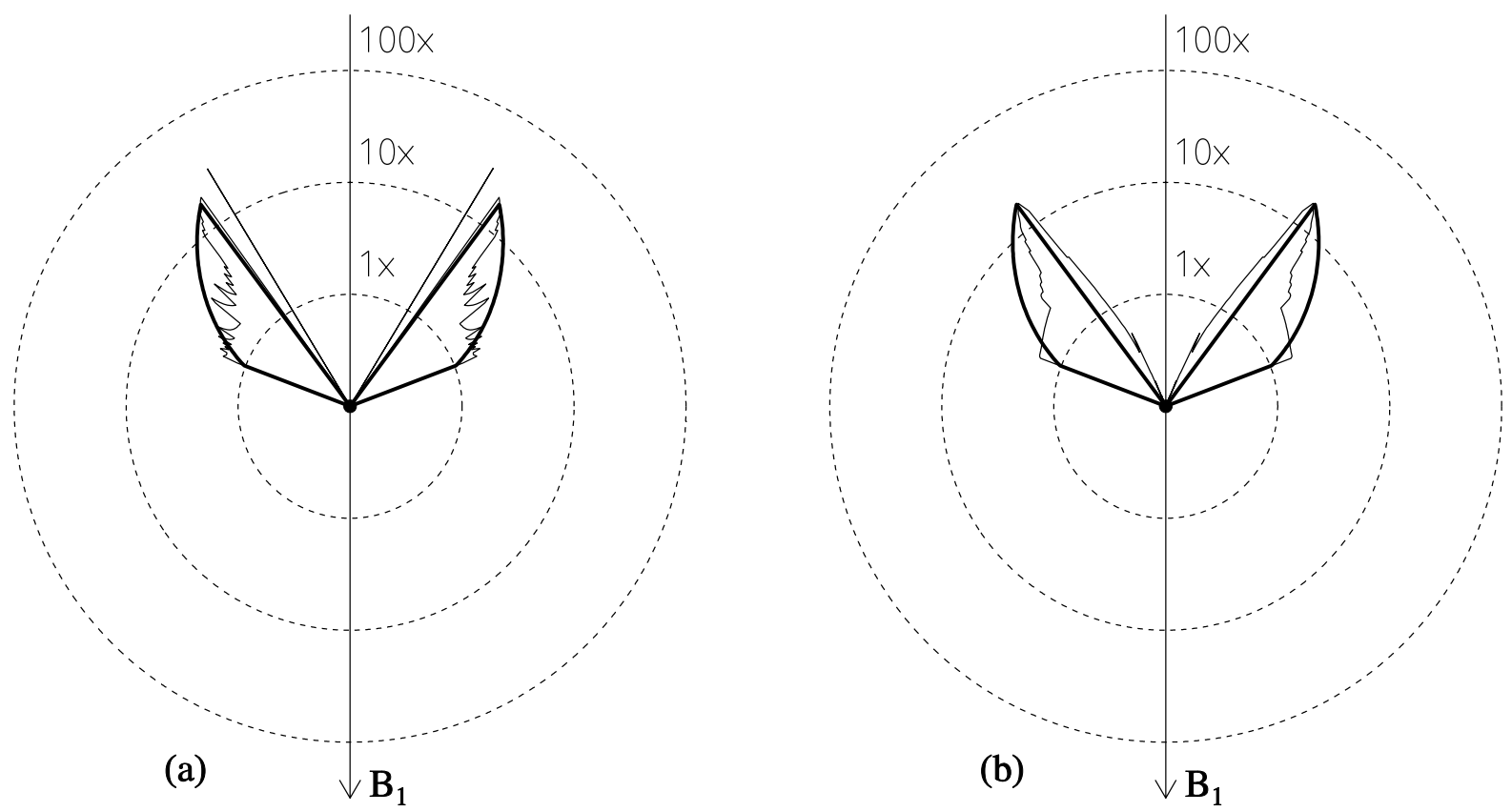

Fig. 11. a) Angular distribution function of accelerated upstream electrons with energy of $3 \mathrm{keV}$ for a wavy shock with $\theta_{B}=85^{\circ}$ in an arbitrarily chosen upstream point (thin solid line). The figure complements the previous Fig. 10. The thick line shows the distribution of reflected electrons at a smoothed shock wave with $\theta_{B_{n}}=\theta_{B}$. The shock front is located in the direction of $\boldsymbol{B}_{1}$. b) The same as in a), but the thin solid line shows the angular distribution averaged along the shock front.

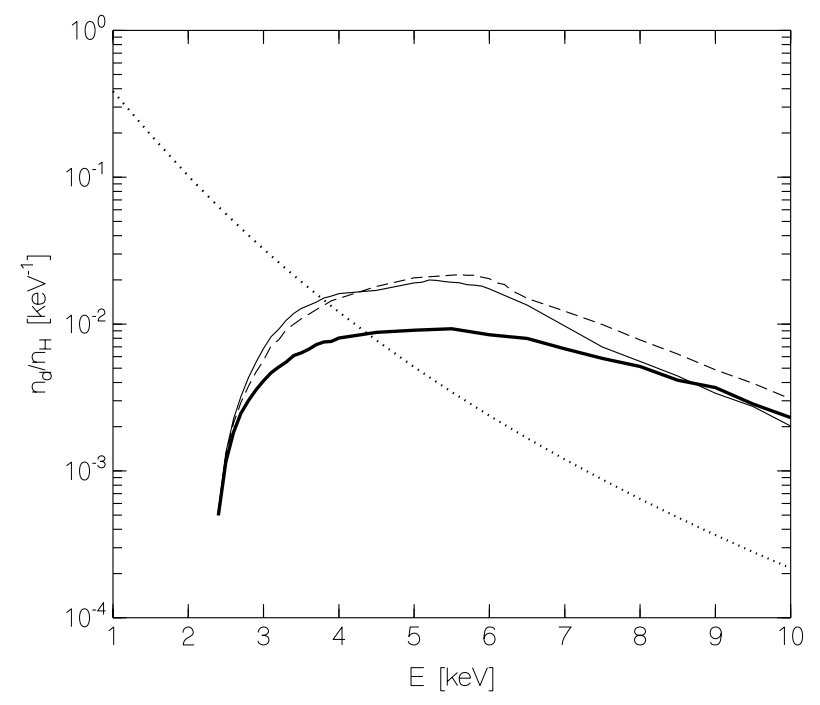

Fig. 12. Differential density of accelerated upstream electrons for a wavy shock with $\theta_{B}=88^{\circ}$. The dotted line shows seed population (initial distribution function (19)), the thick line is for a corresponding smoothed shock, the thin solid and dashed lines are for a wavy shock with $A / L=0.1$ and 0.2 , respectively. The differential density was averaged along the shock front.

It is obvious than in a real situation angular distributions will not be so spiky owing to diffusion processes (like pitch angle scattering) in the shock layer and around it, but it is not probable that they would be wiped out completely. Figure $9 \mathrm{~b}$ shows the same distribution function as in Fig. 9a, but averaged over $Z_{f}$ between 0 and $L$. We see that the averaged distribution function is similar to the distribution function at a corresponding plane shock.

Figures 10 and 11 display angular distributions for a nearly perpendicular wavy shock with $\theta_{B}=85^{\circ}$ in the downstream

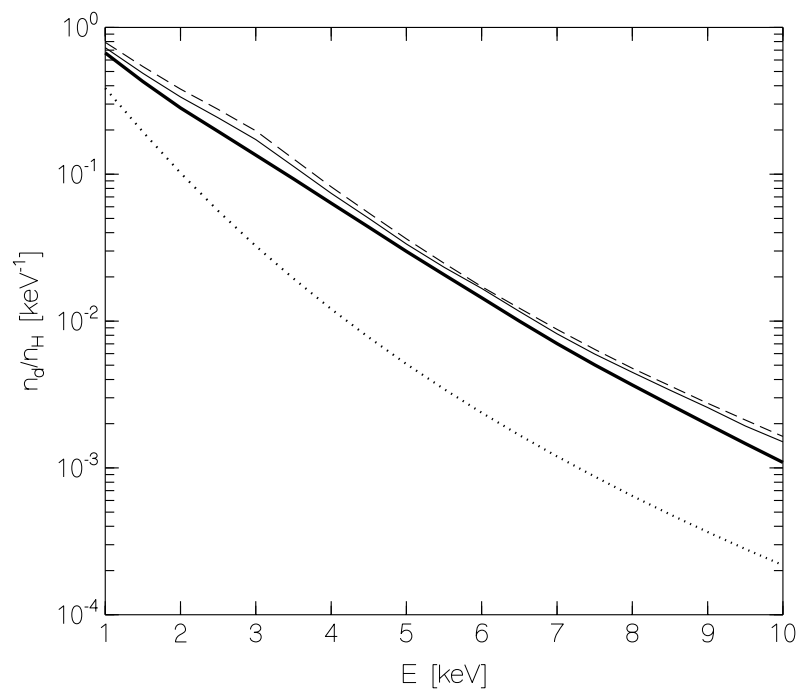

Fig. 13. Differential density of accelerated downstream electrons for a wavy shock with $\theta_{B}=88^{\circ}$. The layout is similar to Fig. 15 . The differential density was averaged along the shock front.

(globally transmitted) and upstream (globally reflected) regions, respectively. The situation is analogous to the perpendicular shock discussed above. Distribution functions of reflected and transmitted electrons are spiky, and the averaged angular distribution functions are similar to the distribution functions at a corresponding plane shock.

The final comparison between acceleration efficiency at a wavy shock and a corresponding smoothed shock wave is shown in Figs. 12 and 13, plotting differential density $n_{\mathrm{d}}$ of accelerated electrons calculated in the upstream and downstream regions, respectively. It was averaged along the shock front. The differential 
M. Vandas and M. Karlický: Electron acceleration in a wavy shock front

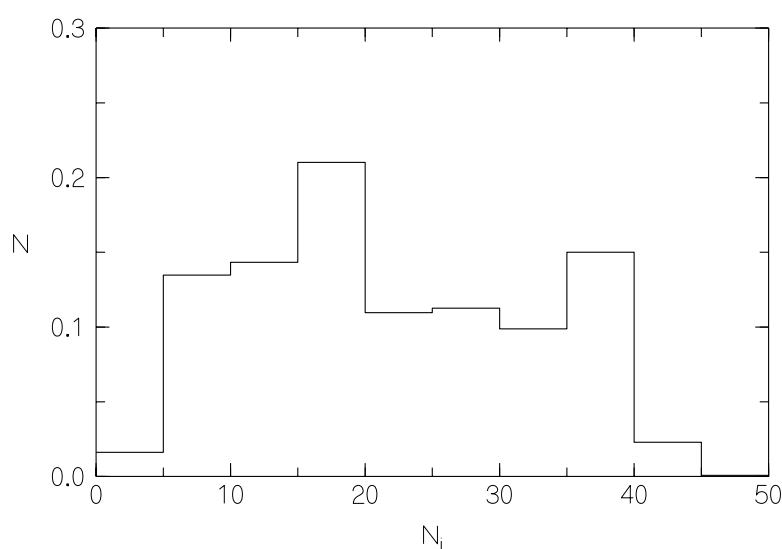

(a)

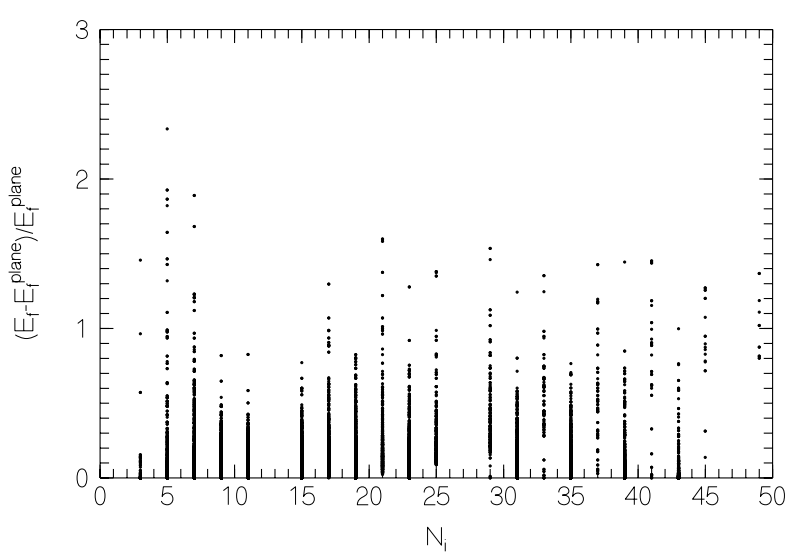

(b)

Fig. 14. a) Histogram showing a distribution of the number $N_{i}$ of interactions of electrons with the shock. $N$ is unitless and represents a fraction. More explanation is in the text. b) Relative energy gain of electrons at the wavy shock over one at a smoothed shock versus the number $N_{i}$ of interactions. $E_{f}$ and $E_{f}^{\text {plane }}$ are final energies after interactions with the wavy shock and a smoothed shock, respectively.

density is defined as

$n_{\mathrm{d}}=\frac{\mathrm{d} n}{\mathrm{~d} E}=\frac{2 \pi}{m} \int_{-1}^{1} f(v, \alpha) v \mathrm{~d} \mu$,

where $\mu=\cos \alpha$ and $n$ is the density,

$n=2 \pi \int_{0}^{\infty} \int_{0}^{\pi} f(v, \alpha) v^{2} \sin \alpha \mathrm{d} \alpha \mathrm{d} v$.

Looking at energetic tails, we see that accelerated electrons at the wavy shock have about one order of magnitude higher differential density than the seed population (initial distribution function), but this density is comparable to the one at a smoothed shock.

Complex trajectories and many interactions of electrons with the shock (e.g., Fig. 8) lead to a question of a stochastic description of electron acceleration at a wavy shock and an application of a Fokker-Planck type equation to this process. We calculated trajectories of about 69 thousand electrons with initial energies $0.5-5 \mathrm{keV}$ and evenly distributed pitch angles and positions along the shock, which had our nominal values with
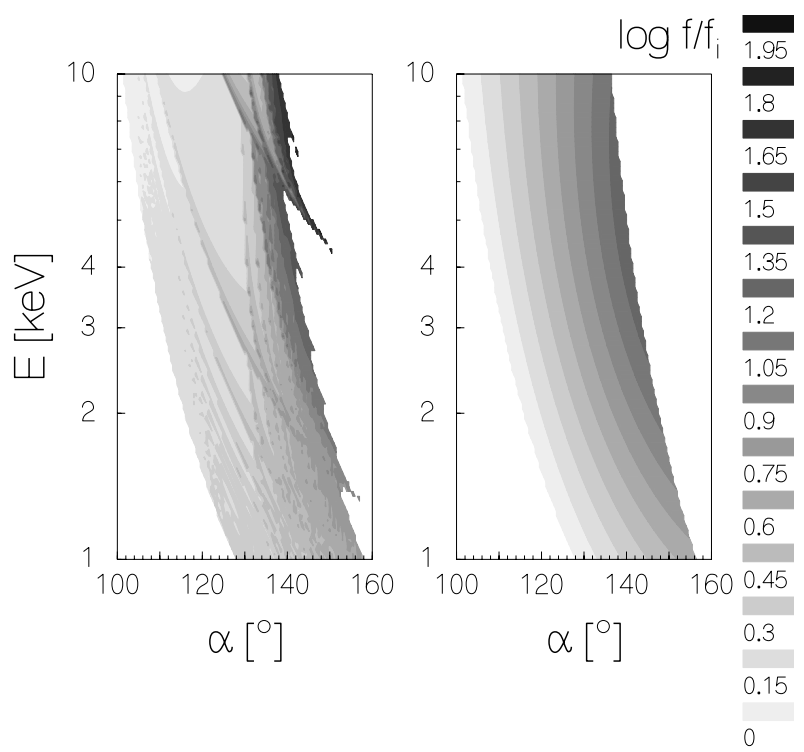

Fig. 15. Contour plot of the normalized distribution function $f(v, \alpha) / f_{i}(v)$ of accelerated upstream electrons as a function of energy $E=\frac{1}{2} m v^{2}$ (in logarithmic scale) and pitch angle $\alpha$ for (left) a wavy shock with $\theta_{B}=85^{\circ}$ and (right) a smoothed shock wave with $\theta_{B_{\mathrm{n}}}=\theta_{B}$. (Plots in Fig. 11a could be constructed from values of the horizontal cut at energy of $3 \mathrm{keV}$.)

$\theta_{B}=90^{\circ}$. Results are summarized in Fig. 14. Figure 14a shows a histogram of the number $N_{i}$ of interactions of electrons with the shock, where $N$ is the fraction from the total number $\Sigma N_{i}$ of interactions. The distribution is rather flat with number of interactions in the tens. Figure $14 \mathrm{~b}$ displays a scatter plot of the number $N_{i}$ of interactions versus the total energy gain $E_{f}$ relatively over the energy gain $E_{f}^{\text {plane }}$ at a smoothed shock. For a stochastic process described by the Fokker-Planck equation, one would expect that energy gains are small for each interaction, and the electron energy increases with the number of interactions (because electrons gain energy at each interaction). But it is not the case, because Fig. 14b does not show such a dependence. In fact, a significant amount of energy electrons gain by one or a few interactions (reflections, cf. Fig. 8). Therefore the Fokker-Planck description is not applicable here.

\section{Discussion and conclusions}

We studied the interactions of electrons with a nearly perpendicular wavy shock front. The present analysis is a continuation of our previous Papers I and II. We found that energy gains of electrons did not depend significantly on the shock thickness, magnetic field profile inside the shock, and shock wavy form; on average, they mainly depended on the angle between the smoothed shock front and ambient magnetic field. It means that, on average, energy gains of electrons at a wavy shock front and a corresponding smoothed shock are comparable. However, we also found that energy gain significantly exceeds an average level for some combinations of initial parameters. One can speculate, that such a repetition of favourable initial conditions could cause a fine structure of type II burst (called herringbones), apparently created by repeated electron beams. Distribution functions of accelerated electrons have a patchy structure (Figs. 15, 16), which changes with position (and time if the wavy front is non-stationary). Such a structure is prone to induce plasma instabilities that will generate plasma waves. These waves may 


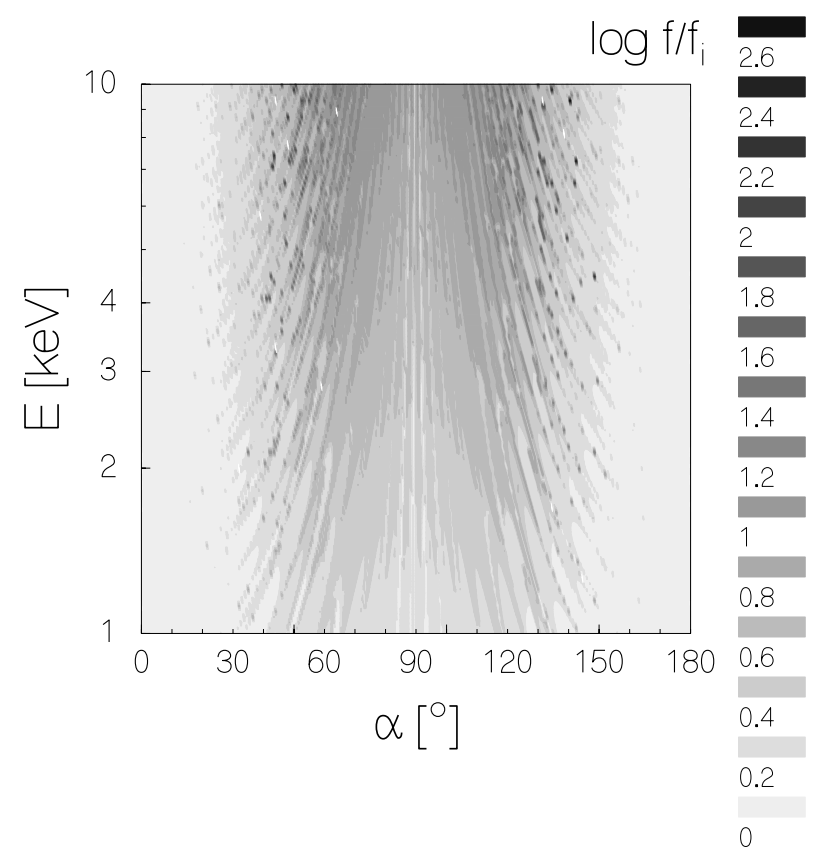

Fig. 16. Contour plot of the normalized distribution function $f(v, \alpha) / f_{i}(v)$ of accelerated downstream electrons for a wavy perpendicular shock. (Plot in Fig. 9a could be constructed from values of the horizontal cut at energy of $3 \mathrm{keV}$.) participate in further acceleration of a fraction of electrons and possibly help to form a herringbone structure.

Acknowledgements. This research was supported by grants 205/09/0170 and P209/10/1680 of the Grant Agency of the Czech Republic, by grant IAA300030701 of the Academy of Sciences of the Czech Republic, and by the MŠMT ČR project ME09009. The research leading to these results has also received funding from the European Commission's Seventh Framework Programme (FP7/2007-2013) under the grant agreement SWIFF (project No. 263340, www. swiff.eu).

\section{References}

Decker, R. B. 1990, J. Geophys. Res., 95, 11993

Gedalin, M. 2001, J. Geophys. Res., 106, 21645

Holman, G. D., \& Pesses, M. E. 1983, ApJ, 267, 837

Knock, S. A., Cairns, I. H., Robinson, P. A., \& Kuncic, Z. 2003, J. Geophys. Res., 108, 1126

Krauss-Varban, D., \& Wu, C. S. 1989, J. Geophys. Res., 94, 15367

Leroy, M., \& Mangeney, A. 1984, Ann. Geophys., 2, 449

Vandas, M. 1989, Bull. Astron. Inst. Czech., 40, 189

Vandas, M. 1991, Bull. Astron. Inst. Czech., 42, 170

Vandas, M. 1995, J. Geophys. Res., 100, 23499

Vandas, M., \& Karlický, M. 2000, Sol. Phys., 197, 85

Vandas, M., \& Karlický, M. 2005, Proc. Solar Wind 11 - SOHO 16,

Connecting Sun and Heliosphere, ESA SP-592, 453

Wu, C. 1984, J. Geophys. Res., 89, 8857

Zlobec, P., Messerotti, M., Karlický, M., \& Urbarz H. 1993, Sol. Phys., 144, 373 